\title{
Superparamagnetic Iron Oxide Magnetic Resonance Imaging
}

National Cancer Institute

\section{Source}

National Cancer Institute. Superparamagnetic Iron Oxide Magnetic Resonance Imaging.

NCI Thesaurus. Code C116461.

A magnetic resonance imaging technique that uses superparamagnetic iron oxide particles as a contrast agent to aid in the identification of multiple disorders, including atherosclerosis, blood-brain barrier dysfunction, and cancer. 